\title{
ANGelika RedDeR
}

\section{Transnationale Germanistik}

\section{Namen als Programm}

„Germanistik - eine deutsche Wissenschaft?““ fragte Eberhard Lämmert 1967 kritisch. Und nun wird hier ein nahezu zweihundertjähriger Disziplinenname mit einem neuen Attribut versehen - wozu und weshalb mit diesem?

„Wenn die Germanistik versuchen soll und will, sich in den europäischen Kontext einzubringen, dann wird es, denke ich, erforderlich, über ihre Entgrenzung ernsthaft nachzudenken, und dafür kommt meinem unmittelbaren Arbeitsbereich, dem Deutschen als Fremdsprache, möglicherweise auch für die wissenssoziologische Struktur eine gewisse Bedeutung, ein eigener Stellenwert zu, den unser Fach, so scheint mir, bisher noch nicht recht ernstgenommen hat, der aber andererseits wohl auch von der Germanistik her auf einige Abwehr stoßen würde."

- so äußerte sich Konrad Ehlich (2000, S. 193 f.) auf der Tagung „EuroDeutsch" an der Ev. Akademie Tutzing 1999, auf welche auch die breit zirkulierten sprachpolitischen „Tutzinger Thesen“ (2000) zurückgehen, denen später die „Homburger Empfehlungen“ (2001) und schließlich das „Mannheimer Memorandum" (2002) folgten.

Bei Ehlich ist von einer Entgrenzung der germanistischen Disziplin die Rede. Genau dieser Gedanke soll im Attribut transnational präzisiert werden. Lateinisch „trans" bedeutet bekanntlich ,jenseits, über ... hin(weg), hinaus" (Georges 1988, S. 3178). Mithin wird eine Grenzüberschreitung zum Ausdruck gebracht, welche sich vermöge „national“ als Grenzüberschreitung des Nationalen, genauer: des „Projektes Nation“ (Anderson 1983/ 1988) konkretisiert. Diese Grenzüberschreitung oder eben Entgrenzung ist sogleich genauerhin zu bestimmen.

Zuvor seien jedoch zwei alternative Attribuierungen erwogen.

Zum einen würde sich ,international" anbieten, also: ,internationale Germanistik“. Dieser Name hätte für sich die Perspektive des ,inter“ als ,innerhalb, zwischen" - zwischen den Nationen eben; und er wäre parallel zu „Interdisziplinarität“" und "Interkulturalität" gebildet, welche zugleich zwei sinnvolle Charakteristika dieser neuen Disziplin darstellen mögen. Allerdings würde diese Entgrenzung als ,internationale Disziplin“ sie zugleich in einen rein politischen Handlungssektor stellen und internationales wissen- 
schaftspolitisches Handeln nahelegen, das den Bestand von Nationen als staatspolitischen Basiseinheiten voraussetzt.

Zum anderen würde sich das Attribut ,postnational “ anbieten, also: ,postnationale Germanistik". Abgesehen von dem unangenehmen Beigeschmack des mit seiner Nachgeschichte sodann Erledigten sowie abgesehen von der assoziativen Verknüpfung mit ihrerseits bereits konjunkturvergessenen Theorie- und Methoden-Moden ( $>$ Postmoderne, Poststrukturalismus), reduzierte sich die Entgrenzung so auf eine bloße Nach-Folge, eine Hinter-Lassenschaft, gar Nachlassverwaltung. Das kann und soll keineswegs gemeint sein.

Es soll auch nicht ,alter Wein in neuen Schläuchen“ präsentiert werden. Vielmehr geht es um eine gesellschaftlich verantwortliche und wissenschaftlich wirksame Konzeption der Gesamtdisziplin nach vorn, von der Gegenwart auf Zukunft hin. In der Sache bedeutet das: Es geht um eine kritische Aufhebung der Germanistik in einer neuen Qualität dieser Wissenschaft.

\section{Kleiner Rückblick auf die Wissenschaftsgeschichte}

Wie kaum eine andere Disziplin ist die Germanistik an die Nation gebunden, und das heißt gemäß Anderson (1988, S. 15): an eine ,vorgestellte politische Gemeinschaft - vorgestellt als begrenzt und souverän". Bereits im 18. Jh., als der Name Germanistik noch Juristen und Historiker neben Philologen umfasste, prägnant dann seit Beginn des 19. Jh. und somit seit der Etablierung der Germanistik im Sinne von Jacob und Wilhelm Grimm, bestand der Fluchtpunkt des wissenschaftlichen Bestrebens in einer politischen Einheit bürgerlichen Zuschnitts. Dieser Fluchtpunkt war vor allem durch den Konnex von „Nation und Sprache“ hergestellt - so auch der Titel des exzellenten Sammelbandes von Andreas Gardt (2000).

Angesichts einer fehlenden politischen Einheit (a) am Ende des „Heiligen Römischen Reichs Deutscher Nation“ 1806, (b) während der „Freiheitskriege“ gegen Napoleon 1814/15, die lediglich in einer Festlegung des Territoriums des Deutschen Bundes von 39 Staaten unter österreichischer Hoheit auf dem Wiener Kongress endeten und (c) durch das Scheitern der Revolution von 1848 und der Frankfurter Paulskirche, bot sich einzig das Konzept einer Sprachnation als vorgestellte Gemeinschaft an.

Das gut zweihundertjährige Bemühen um eine deutsche Hochsprache sowie die Entwicklung des Deutschen zu einer Literatursprache und vor allem zu einer eigenen Wissenschaftssprache war zu dieser Zeit auf seinen Höhepunkt gekommen und bot einen handlungspraktischen Fundus, der in allen Praxisbereichen funktional war. Aufgrund der ausgleichenden Rückbindung an die Alltagssprache war dieser deutschen Hochsprache seit der Reformation zugleich ein prinzipiell demokratisches Moment eigen, das bei geeigneter schulischer Deutschlehre faktisch allen Mitgliedern der Sprachgemeinschaft ohngeachtet ihrer sozialen Zugehörigkeit Zugänglichkeit ermöglichte. Preußen ergriff mit seinen Bildungsreformen als erstes Bun- 
desland diese weite sprachliche Chance und sicherte durch die Einführung des Staatsbeamtentums zugleich „,nationale“ Loyalität und Unabhängigkeit der Lehrenden von Kirche und Ökonomie - drei Errungenschaften, die wir derzeit in der BRD aufs Spiel zu setzen uns anzuschicken scheinen.

Die Ambivalenzen, Bruchlinien und deutschtümelnden bis reaktionären Ausschöpfungen der Potentiale innerhalb der Germanistik - schon bei der genetischen Sprachforschung und forcierten Volkssprachbewegung der Grimms - sind sorgfältig eruiert und dargestellt worden; exemplarisch verweise ich neben Gardt (1999) auf Müller (1974/repr. 2000) und Janota (1980) sowie Rompeltien (1994). Erinnert sei lediglich an drei Umschlagpunkte.

(1) Mit der politischen Nationenbildung 1870/71 verschob sich das Konzept von einer Sprachnation zur Kulturnation, und zwar im wesentlichen auf der Basis des bildungsbürgerlichen Kulturprotestantismus. In dieser Phase wurde die Neuere Deutsche Literaturwissenschaft vor allem in Form der Literaturgeschichte neben Mediävistik und historischer Sprachwissenschaft in die Germanistik integriert und Philologie zum hauptsächlichen methodischen Ausweis der Gesamtdisziplin.

(2) Mit dem Nationalsozialismus wurden nationalistische Unterströmungen in der Germanistik (wie auch in anderen Wissenschaften) politisch „hoffähig“ und rassistische Begründungszusammenhänge für Nationenbildungen - vor allem seitens der germanistischen und indogermanistischen Linguistik (Römer 1985) - imperialistisch umgesetzt sowie als Legitimationsausweis für Barbarei genutzt. Widerstände in der Disziplin wurden disziplinarrechtlich mundtot gemacht, in die Emigration getrieben oder gar im physischen Sinne vernichtet (Maas 1996).

(3) An die Gründung der beiden Staaten DDR und BRD knüpfen sich jeweils Hoffnungen auf einen disziplinären Neubeginn unter different aufgeklärten bildungspolitischen Vorzeichen und wiederum nationalem Einheitstrachten, teils sprachlich, teils nationalhistorisch begründet. Betrachten wir die alte Bundesrepublik kurz genauer.

In der BRD verschoben sich die Gewichte hin zu einer modernen, von der philologischen Tradition emanzipierten germanistischen Linguistik sowie einer stärker theoretischen NdL, während die Mediävistik mehr und mehr in die Defensive geriet. Die nationale Bindung der Disziplin blieb im großen und ganzen bestehen, ja gewann unter den Vorzeichen eines freiheitlich-demokratischen Rechtsstaates besonders in der Bildungsreform der siebziger Jahre einen Schub nach vorn in Richtung auf kritisch emanzipierte Staatsbürgerlichkeit im Wege chancengleicher Bildung in muttersprachlicher Kommunikation und Textreflexion.

Seit 1989 bzw. 1991 besteht eine völlig neue Konstellation: Mit der erweiterten BRD ist eine nationale Einheit hergestellt. Mit dem Wegbrechen der Sowjetunion ging das politische Gegengewicht gegen imperiale, ja ,globale" Weltmarktansprüche unter der Hegemonie eines einzelnen föderativen Nationalstaates verloren. Andererseits ist mit der Realisierung einer überna- 
tionalen europäischen Einheit in Form der EU eine neue Herausforderung verknüpft.

Für die Germanistik kann das zweierlei bedeuten:

- Entweder verliert sie aufgrund ihrer nationalen Bindung ihren historischgesellschaftlichen ,Sitz im Leben" und transformiert sich zur orchideenhaften Wissenschaft eines historischen Erbes, zumal dann, wenn eine globale lingua franca auch den europäischen Alltag monolingual dominiert. Die Halbierung der sprachwissenschaftlichen Lehramtsausbildung und Umgewichtung auf literaturwissenschaftliche, ja literaturgeschichtliche Ausbildung einzig für Deutsch in den soeben verabschiedeten Rahmenrichtlinien von Baden-Württemberg deuten eine derartige landespolitische Erwartung an.

- Oder die Germanistik innoviert sich, indem sie transnationale Dimensionen, die sie durchaus längst enthält, zur Geltung bringt, kritisch weiterentwickelt und in ein interdisziplinäres Selbstverständnis aktiv und selbstbewusst einbringt.

- Wie könnte das aussehen?

\section{Systematische Aspekte einer Transnationalen Germanistik am Beispiel der Linguistik}

Man wird nicht erwarten, dass ich im folgenden eine Transnationale Germanistik in allen ihren Dimensionen gleichermaßen detailliert entwerfe. Vielmehr möchte ich exemplarisch vorgehen und mich besonders auf die linguistischen Anteile konzentrieren. Dabei werden allerdings Bezüge zu literaturwissenschaftlichen und mediävistischen Anteilen deutlich werden und bestimmte Facetten hervorgehoben. Dies ist nicht zuletzt darin begründet, dass sich nach meiner Auffassung auch die drei klassischen germanistischen Teilgebiete wieder in einer engeren Verzahnung kooperativ zusammenfinden sollten, statt konkurrenziell auseinanderzudriften. All das hat freilich auch Konsequenzen für eine transnational germanistische Didaktik, welche allerdings eigens zu thematisieren wären (s. Durell und Krumm in diesem Band).

Eine Reihe von Aspekten gilt es, für das Konzept einer transnationalen Germanistik generell zu berücksichtigen und für die transnational-germanistische Linguistik im besonderen zu verfolgen. Sie sind vor allem grundlagentheoretischer, methodologischer und sprachtheoretischer wie wissenschaftssystematischer und schließlich wissenschaftsorganisatorischer Natur. Ich möchte mich angesichts der fortgeschrittenen Zeit und Tagungsmüdigkeit lediglich auf drei Fragen konzentrieren, die mir zentral zu sein scheinen:

1) Wie kann eine Systematisierung oder Theoretisierung der unterschiedlichen Phänomene des Deutschen aussehen, wenn diese Einzelsprache nicht als Nationalsprache gefasst wird?

2) Welche linguistischen Gegenstandsbereiche sind für eine transnationale Germanistik besonders relevant? 
3) Wie kann ein Wechselverhältnis der Forschungen und Einsichten, die in der „Auslandsgermanistik" und der „Inlandsgermanistik" sowie in „Deutsch als Fremd- und als Zweitsprache“ gewonnenen werden, institutionell verankert und befördert werden?

Ich begebe mich mit dem - selbstverständlich selektiven - Versuch, auf diese Fragen einzugehen, freilich auf höchst gefährliches Glatteis einer zuweilen provokanten Fachkritik. Ich nehme das in Kauf, um in eben diesem Fach eine kritische und m.E. doch existentiell notwendige Diskussion anzustoßen, die hoffentlich Fairness des wissenschaftlichen Streitens walten lässt. Dass ich nicht fachblind und also in den Aspekten von unschuldiger Desinteressiertheit gefangen bin, wird man mir, so hoffe ich, zugestehen. Ich kann mich dabei zugleich auf die Beiträge und Podiumsdiskussion zur Sektion 3 „Entnationalisierung II“ des Deutschen Germanistentages 2001 beziehen (Kugler et al. im Druck).

\subsection{Zur Systematisierung und Theoretisierung}

„Sprache als System versus Sprache als Handeln“ - so lässt sich mit Artikel 67 im HSK „Sprachphilosophie“ (Ehlich 1996) die grundlagentheoretische Alternative beschreiben, die für alle Sprachwissenschaften seit der strukturalistischen Wende zu Beginn des 20. Jahrhunderts und der pragmatischen Wende seit den siebziger Jahren ungebrochen virulent ist. Bei näherer Betrachtung entpuppt sich ein Teil dieser Alternative als Schein, insofern nämlich auch eine Handlungstheorie von Sprache allein auf die analytische Rekonstruktion einer systematischen Sprachstruktur abzweckt. Dies geht, weil das sprachliche Handeln selbst systematisch und strukturiert ist. Der Unterschied besteht in der Fundierungskategorie und in einer additiven versus integrativen Konzeption von Sprach-Gebrauch oder Pragmatik.

Der Systembegriff von Sprache bzw. der sprachlichen Struktur ist grundsätzlich frei von systematischen Bestimmungsmomenten wie Sprecher oder gar Hörer, von sprachbezogenen mentalen Tätigkeiten und von historischgesellschaftlicher Praxis. Dies gilt auch dann, wenn das System theoretisch einem abstrakten Sprecher/Hörer als Kompetenz oder ,i-language“ im kognitivistischen Sinne zugeschrieben wird. Die theoretische Abstraktion kann so zur Reduktion des Gegenstands ,Sprache“ in einem Ausmaß geraten, das an einen tendenziellen Verlust heranreicht - ich erinnere diesbezüglich an die polemischen Auseinandersetzung zwischen Ludwig Jäger einerseits und Manfred Bierwisch sowie Günther Grewendorf andererseits in der Zeitschrift für Sprachwissenschaft 1993.

Die Freiheit des reinen Sprachsystems ist m. E. erkauft um den Preis beliebiger Addierbarkeit oder auch Reduzierbarkeit; und sie erlaubt problemlos ideologische Funktionalisierung, etwa in Form von Sprachbewertung aus nationalsprachlicher Sicht. Diejenigen, die von dem einzelsprachlichen System (,la langue“) in der addierten ,parole“ Gebrauch machen, gelten dann 
z.B. als die „Sprach-Teilhaber" (sic) ${ }^{1}$ oder Staatsbürger der jeweiligen Nationalsprache bzw. als Subgruppen davon. Ihr konkretes sprachliches Handeln bleibt gegenüber dem Sprachsystem als fait social bzw. fait accompli äußerlich. Leicht stellt sich so ein normatives und regulatives Denken über Sprache ein, das beispielsweise für Fragen der Einbürgerung fatale Konsequenzen haben kann.

Bei einem Handlungsbegriff von Sprache bildet die Kommunikation als verbale Interaktion zwischen einem Sprecher und einem davon verschiedenen Hörer mit ihren jeweiligen aktionalen und mentalen Handlungspotentialen ein integrales Bestimmungsmoment des Begriffs - zudem die „Sprechsituation“, in der die Kommunikation aktual wird. Insofern stellt der Handlungsbegriff von Sprache eine historisch-gesellschaftliche Kategorie dar, die aber eben darum nicht an eine bestimmte Gesellschaftsformation gebunden werden kann. Vielmehr fordert er dazu auf, die Sprachstruktur im Wechselverhältnis zur besonderen historischen Gesellschaftsform analytisch herauszuarbeiten und so Kontinuitäten und Diskontinuitäten zu erfassen.

Genau dies aber dürfte für eine transnationale Konzeption einer Einzellinguistik wie der germanistischen Linguistik gut geeignet sein. Denn eine historisch-gesellschaftlich veränderte, neue Gesellschaftsformation, die beispielsweise nicht mehr oder nicht primär national organisiert ist, zeitigt zugleich einen Wandel des Handlungsmittels Sprache - des Handlungsmittels Deutsch im besonderen, das zu erforschen wir aufgerufen sind. Die gesellschaftliche Fundierung des Handlungssystems Sprache steht zugleich in einem kritischen Verhältnis zu individuell basierten Konzepten, denen gemäß etwa - wie bei Coulmas (1999) - die Einzelsprachen einer freien Wahl des Sprechers anheimgestellt sind und also kommunikativ wie kognitiv beliebig werden.

Wissenschaftsgeschichtlich ließen sich - neben Austins Sprechhandlungstheorie - Anknüpfungspunkte an Karl Bühlers Gedanken von „Sprachwerk“ und ,Sprachgebilde“, an Philipp Wegeners Ausführungen zur sprachpsychologisch basierten, funktionalen Sprachstruktur und schließlich an Wilhelm von Humboldts Konzept von ,ergon" und ,energeia" in pragmatisch aufgeklärter, nicht-idealistischer Weise nutzen (vgl. Knobloch 1988). Humboldt formuliert in Ueber die Verschiedenheit des menschlichen Sprachbaues und ihren Einfluss auf die geistige Entwicklung des Menschengeschlechts: „Um daher verschiedne Sprachen in bezug auf ihren charakteristischen Bau fruchtbar miteinander zu vergleichen, muss man der Form einer jeden derselben sorgfältig nachforschen und sich auf diese Weise vergewissern, auf welche Art jede die hauptsächlichen Fragen löst, welche aller Spracherzeugung als Aufgaben vorliegen." (1963, Bd. III, S. 417)

${ }^{1}$ Gerhard Stickel nutzte in seiner Einladung zu dieser Tagung - freilich per Anspielung und mit dem klaren Zeichen der Distanzierung - die Metapher vom „Eigentümer“" der deutschen Sprache und Literatur. 
Neue Aufgaben, die sich systematisch den auf Deutsch sprachlich Handelnden in einem transnationalen Europa und einer multikulturellen Gesellschaft stellen, würden somit - funktional-pragmatisch gesprochen - als Problemlösungen für sprachexterne Zwecke durch bestimmte neue Mittelstrukturen ihren Niederschlag finden, wobei die bereits vorhandenen sprachtypischen Form-Funktionsrelationen sowie die bewährten Mittel für sprachinterne Zwecke eine bestimmte Drift der Veränderungswege vorgeben (Ehlich 1981). Weder puristische noch formalistische Verdikte intervenierten zum Behufe des Erhaltes der Nationalsprache in einer bestimmten Form. Wohl aber würde die Fortentwicklung einer vollausgebauten Sprache wie des Deutschen für alle gesellschaftlichen Praxisbereiche als Verantwortung der sprachlich Handelnden und also als praktische sprachpolitische Herausforderung ins Bewusstsein treten - allemal eine Aufgabe für den - mit dem unvergessenen Pierre Bourdieu gesprochen - ,homo academicus“.

Jedenfalls lässt sich ohne weitere grundlagentheoretische Erwägungen resümierend sagen: Die Historizität von Sprache sollte in der transnationalen Germanistik als gesellschaftliche eine Behandlung finden. Der gewählte Sprachbegriff müsste das begünstigen.

Pragmatische Theorien des Sprachwandels oder Forschungen zur historischen Pragmatik etwa durch (alphabetisch genannt) Dieter Cherubim, Helmut Henne, Peter von Polenz oder Joachim Schildt und eine - wie Oskar Reichmann unlängst hervorhebt (2001) - „europäische Sprachgeschichtsschreibung" sowie eine Sprachgeschichte DaF, die Helmut Glück derzeit vorantreibt, bieten sich dafür unmittelbar an. Zugleich sind empirische Forschungen zur Kommunikation in der Gegenwart dazu nötig.

Eine andere Grundlage germanistischer Linguistik wäre aus transnationaler Sicht neu zu bedenken.

Bislang geschehen die Forschungen - begünstigt durch die bildungspolitische Kontinuität seit dem Kaiserreich und scheinbare Homogenität der bürgerlichen Gesellschaft sowie durch den kolonialen Misserfolg Deutschlands - zumeist in Kategorien der Einsprachigkeit oder Monolingualität. Zwar hat die Soziolinguistik bzw. Sprachsoziologie der späten sechziger Jahre die Homogenitätsunterstellung nachhaltig gesprengt und mit dem Konzept der sprachlichen Varietät auch der Vielfalt des Deutschen Rechnung zu tragen versucht. Doch drohten darüber bald die differenten funktionalen Reichweiten zugunsten einer wertenden Egalisierung aus dem Blick zu geraten - etwa nach dem Motto: gleiches Bildungsrecht für Dialekte bzw. Soziolekte und Hochsprache -, so dass ein gewisser sprachpolitischer Defätismus hinsichtlich hochsprachlicher Konkurrenzen oder gar Hegemoniebestrebungen eintreten konnte. Die jeweiligen sprachpolitischen Argumentationslinien von Ulrich Ammon scheinen mir dafür nicht untypisch zu sein.

Erst seit der intensiveren Befassung mit Bilingualität, ja mit Mehrsprachigkeit ist eine neue Sicht und Wertung der komplexen sprachsoziologischen Phänomene möglich geworden. Code-switching und Mischsprachen 
erfahren eine systematische Analyse - nicht zuletzt hier in Mannheim (ich verweise nur auf Rosemarie Tracy (z. B. 2000), Inken Keim und Werner Kallmeyer (z. B. 1994)) und der Spracherwerb stellt sich, wie z. B. die Projekte im Hamburger SFB „Mehrsprachigkeit" zeigen, in Kategorien der Mehrsprachigkeit sehr viel differenzierter und sprachpsychologisch wie entwicklungspsychologisch komplexer dar als unter der meist monolingualen Perspektive des Erstspracherwerbs. Kategorien wie „Muttersprache“ erfahren eine erneute Kritik; im Falle simultaner statt sukzessiver Mehrsprachigkeit ist von „mehreren Erstsprachen" die Rede. Ob die Theoretisierung nun in Kategorien der Parameterwechsel (Meisel 2000; N. Müller 2001) oder in funktional-pragmatischen Kategorien (Rehbein/Grießhaber 1996; Garlin 2000 ) erfolgreicher verläuft, wird die weitere Forschung zeigen.

Für eine transnational-germanistische Linguistik erscheint mir ein Denken in Kategorien der Mehrsprachigkeit allerdings erforderlich. Kollege Sasalatti (in diesem Band) hat uns auf dieser Tagung in faszinierender Weise die mehrsprachige Wirklichkeit Indiens vor Augen geführt; man hätte Ähnliches aus afrikanischen Ländern präsentieren können. Die heutige Realität in den europäischen Nationen, mehr noch die angestrebte und für alle Mitgliedsstaaten zu befürwortende Mehrsprachigkeitspolitik in Europa unterstützen dies - aber auch ein sprachtheoretischer Impetus, der grundsätzlich sprachbezogene mentale Prozesse einbezieht und auf eine sprachvergleichend angelegte Strukturanalyse abzielt, freilich auf keine solche, die genetisch im Grimmschen oder genetisch im modernen biochemischen Sinne ist.

\section{2 $\mathrm{Zu}$ linguistischen Gegenstandsbereichen}

Grundsätzlich sind für eine transnational-germanistische Linguistik alle sprachlichen Gegenstände von Interesse. Allerdings sind jeweils Brechungen über andere Sprachen und damit sorgfältige Reflexionen auf die selbstverständlichen Voraussetzungen des gegenständlichen Zuschnitts wie der analytischen Kategorien gefordert. Wir kennen das Problem aus der Grammatikgeschichte in Form der allmählichen und mühsamen, möglicherweise bis heute nicht abgeschlossenen Emanzipation von den Kategorien der griechisch-lateinischen Tradition und der assertionsbasierten Satzzentriertheit seit Aristoteles.

Spezifisch wird man gewiss auf Anhieb an Forschungen zum Sprachkontakt und empirische Analysen zur interkulturellen Kommunikation denken. Hier ist ein interessantes Wechselverhältnis zwischen Deskription und theoretischer Reflexion zu beobachten - bis hin zu der These, dass Interkulturalität zur Konstruktion neuer eigener Kommunikationsformen, neuer Diskurstypen zu führen vermag (so Koole/ten Thije 1994). Dabei dürfte auch eine Kritik des Kulturbegriffs hinsichtlich seiner wissenschaftlichen Qualität einerseits (Ehlich 1996) und seiner stillschweigend nationalen Bindung andererseits anstehen, wie sie sich etwa in der Gegenüberstellungen von ,interkulturell“ und ,intrakulturell“" (Hinnenkamp 1994) ausdrückt, wie sie bei einer gesellschaftshistorischen Basierung aber auch überwunden werden 
könnte (vgl. Redder/Rehbein 1987). Neben breiten sprachsoziologischen Fragestellungen drängen sich für eine transnationale Wissenschaftsperspektive zudem mentale Prozesse als relevante Gegenstände auf, und zwar sowohl aus wissenssoziologischer Sicht und ausgreifend bis hin zu einer entsprechend untermauerten Semantik, als auch aus sprachpsychologischer Sicht, wobei Erwartungsstrukturen der sprachlich Handelnden an diskursive und textuelle Funktions- und Formzusammenhänge wie auch Leistungen des individuellen und kulturellen Gedächtnisses in den Blick träten.

Ich möchte das Augenmerk nun auf drei eigene Gegenstandsgebiete richten, die teils als solche innovativ sind, teils neu angegangen werden. Ich meine die Interkulturelle Hermeneutik, die Komparatistik der Wissenschaftssprachen und eine kontrastiv angelegte Linguistik, vor allem die Kontrastive Grammatik. Beginnen wir mit Letztgenanntem.

Werner Abraham spricht von der „Faszination der kontrastiven Linguistik $\mathrm{DaF}$ " (in diesem Band). Dies geschieht im Rahmen einer bestimmten linguistischen Theoriebildung, die sich vornehmlich auf die Systematik der sprachlichen Formen und deren innere Funktionen konzentriert, im Sinne eines semiotischen Systemgedankens von Sprache; außerhalb dieses Rahmen Liegendes lässt sich so vereinzelt als Surplus konzedieren.

Ich möchte dem ein komplementäres handlungstheoretisches Projekt zur Seite stellen. Es ist in der am IDS 1997 von Zifonun, Hoffmann und Strecker verantworteten „Grammatik der deutschen Sprache“ programmatisch formuliert und dort in innovativen Gegenstandsbereichen wie der ,Grammatik von Text und Diskurs" (Teil C, L. Hoffmann) bereits angegangen worden. Der theoretischen Kategorie „Kompetenz“ steht dann die mentale Kategorie „Sprachwissen“ gegenüber, und das Tertium comparationis bilden systematisch und präzise bestimmte sprachliche Funktionen und deren Struktur. Hoffmann formuliert das im Jahrbuch DaF (1995, S. 239) prägnant: „Letztlich vergleichen wir die Formen als Ausprägungen einer Funktion, sonst sind sie unvergleichbar". Ob das gegenwärtige IDS-Projekt einer vergleichenden Grammatik diesen fruchtbaren Beginn fortführt und ausbaut, wird sich erweisen; die Ausführungen von Zifonun (in diesem Band) deuten eher auf eine Absetzbewegung hin.

Und weitergehend wird das Ziel einer funktional-grammatischen Komparatistik als eine Rekonstruktion der mentalen Prozesse verbaler Planung und verbalen Verstehens erkennbar: „Wenn wir im Sinne Humboldts [...] eine Kontrastive Grammatik einrichten wollen, sollten wir eben nicht allein äußere Formen von Sprachen miteinander vergleichen, konfrontieren und identifizieren, sondern die mit den Sprachen jeweils verbundenen Handlungen, psychischen Aktivitäten und Denkformen. In einem umfassenden Sinn sind Sprachen Systeme von Kommunikation, die bei der Kontrastierung letztlich miteinander in ein Verhältnis zu setzen sind.“ (Rehbein 1995, S. 265).

Es geht demnach systematisch um das Verhältnis von Grammatik und mentalen Prozessen (vgl. Redder/Rehbein 1999) und im einzelnen etwa um 
die Frage, ob die hörerseitige Mitkonstruktion linear umzukehren ist - bei der Satzverarbeitung etwa vom Türkischen zum Deutschen hin - oder ob das Sprachwissen für das Deutsche auf eine sprachtypologisch wesentliche „Klammerstruktur“ umzuorganisieren ist, wie Weinrich (1986) seit langem betont - motiviert durch den Vergleich der recht nahen indoeuropäischen Sprachen Französisch und Deutsch. Eine solche theoretische Basierung dürfte für produktive wie für rezeptive Grammatiken fruchtbar sein und nicht zuletzt für Fragen von Übersetzung und Dolmetschen Relevanz besitzen (s. Fabricius-Hansen 1999 und in diesem Band).

Mit dem zweitgenannten Gegenstandsbereich, den Wissenschaftssprachen und ihrer Komparatistik, betritt man im wesentlichen ein Neuland, das vor allem am Münchner Institut für Deutsch als Fremdsprache von Harald Weinrich und Konrad Ehlich zu betreiben angeregt wurde.

In Europa bieten sich geradezu optimale Voraussetzungen dazu, da mit dem Griechischen, Lateinischen und Arabischen historisch-gesellschaftliche Sprachleistungen von Weltrang in einem Kulturraum präsent waren, welche einander und eben auch das Deutsche fruchtbar prägten. Und heute bieten sich hier mehrere moderne Wissenschaftssprachen an, z. B. das Französische, Russische und Deutsche sowie Englische, welche gleichermaßen fortzuentwickeln und gemeinsam zu nutzen sich lohnt. Angesichts der Internationalisierung wissenschaftlicher Studien und Forschungen sowie nicht zuletzt angesichts einiger Wissenschafts-Sprach-Politik monolingualer Provenienz hat die transnationale Germanistik hierin einen zentralen Aufgabenbereich.

Ich denke, dass dies kaum bestritten wird. Es ist allerdings eine offene politische und ökonomische Frage, ob dem eine angemessene Förderung bei der Studien- und Wissenschaftsplanung sowie bei den Drittmittelverwaltern beigemessen werden wird.

Empirische Forschungen zur Hochschul- und Forschungskommunikation in verschiedenen Wissenschaftssprachen und Wissenschaftskulturen sowie Forschungen zum Erwerb des Deutschen als fremder Wissenschaftssprache in eigenen Lehr-Lern-Situationen oder aber in der konkreten universitären Studienpraxis (z. B. Wiesmann 1999, Moll 2001, Schramm 2001, Redder 2002) sind von unmittelbarer praktischer Relevanz für Studienerfolge und für die argumentative Einbringung von vielfältigen und mehrperspektivischen Erkenntnissen in den wissenschaftlichen Diskurs. Hierzu sind nachhaltige interdisziplinäre und internationale Anstrengungen erforderlich.

Als letztes möchte ich mit der Interkulturellen Hermeneutik einen Bereich ansprechen, der nicht nur mit den Namen Dietrich Krusche einerseits und Alois Wierlacher andererseits (z. B. 1990) verknüpft sein sollte. Vielmehr stellt er eine offene Herausforderung dar, die sich vor allem auf eine engere Kooperation zwischen einer transnational-germanistischen Linguistik mit einer entsprechenden Literaturwissenschaft und auch Mediävistik richtet.

Eine kulturwissenschaftliche Mediävist hat die europäische Mehrsprachigkeit bzw. Kontaktsituation für die Herausbildung der deutschen Litera- 
tur länger schon im Auge, einschließlich der Umschläge von Mündlichkeit in Schriftlichkeit und der sozialen Funktionen in historisch differenten Produktions- und Rezeptionssituationen. Die eo ipso transnationale literaturwissenschaftliche Komparatistik war wiederum lange einem Konzept von Weltliteratur verpflichtet, das letztlich nur das poetologisch und ästhetisch „Beste“ aus den Nationalliteraturen selegierte und verglich, doch zeichnen sich hier wie auch in der traditionell national orientierten Neueren deutschen Literaturwissenschaft literatursoziologische und literaturtheoretische Neuerungen ab, die für eine interdisziplinäre Zusammenarbeit im engeren Sinne hilfreich sind. So werden heute - wenngleich erst vereinzelt - neben dem nationalen Kanon liegende deutschsprachige Literaturen wie z.B. die Exiloder die Migrantenliteratur ernsthaft zur Kenntnis genommen und gar für eine kritische Reflexion des Literaturbegriffs sowie der literarischen Kommunikation aufgegriffen. Differente literarische Texttraditionen, differente Aneignungsformen von Literatur und ein verschiedener Umgang mit Literatur könnten mit einer linguistisch basierten Untersuchung des Verstehens literarischer Texte sowie einer Analyse der Handlungspotentiale, die in Texten oder theatralischen bzw. cineastischen Diskursen geronnen sind, zu fruchtbaren neuen Einsichten führen. Empirische Forschungen in deutschsprachigen und fremdsprachigen Zusammenhängen legen sich unmittelbar nahe und sind auch teilweise bereits aufgenommen worden.

\subsection{Zum Wechselverhältnis von Außen- und Innensichten}

Zu meinem dritten Unterpunkt ist im Laufe der Tagung bereits Vieles gesagt worden. Ich kann und will dies nicht im einzelnen wieder aufgreifen und in einen Bezug zum Konzept einer Transnationalen Germanistik setzen. Doch scheint mir angesichts der administrativen Bewegung, die in die Bildungspolitik und vor allem in die institutionelle Grundlegung von Wissenschaft in der BRD gebracht wurde und in vielen anderen Ländern ihr Pendant hat, noch einmal ein Blick auf disziplineigene Bewegungen und Verortungen von Lehre und Forschung zu deutscher Sprache, Literatur und Kultur angebracht. Meine Vorschläge berühren sich hier teilweise mit denen von Krumm (in diesem Band).

Das Konzept einer transnationalen Germanistik geht grundsätzlich von der wesentlichen Verschiedenheit und also von einer anerkannten Eigenheit germanistischer Tätigkeiten in verschiedenen Gesellschaften, in verschiedenen Ländern und Weltregionen aus. Es setzt geradezu positiv auf die Vielfalt und die darin einbeschlossene wechselseitige Bereicherung - ganz so, wie Wilhelm von Humboldt dies 1830-35 für die verschiedenen Sprachen der Welt formulierte:

„Gerade in ihrer völkertrennenden Eigenschaft vereinigt sie [die Sprache; A. R.] durch das Wechselverständniss fremdartiger Rede die Verschiedenheit der Individualitäten, ohne ihnen Eintrag zu thun. [...] 
Aber die Sprache im Allgemeinen, die ganze menschliche als Eine genommen, und jede einzelne, welche in diese höhere Berührung kommt, gewinnen, je grösser die Masse der Gegenstände, der in Sprache verwandelten Welt, wird, und je vielfacher die in gemeinsames Verständniss tretenden Individualitaeten, diese eigentlich sprachbildenden Potenzen, sind.“ (1963, S. 150/151)

Wissenschaftssoziologisch und wissenschaftstheoretisch hätte man konsequenterweise eine Komparatistik der germanistischen Wissenschaften anzuregen. Doch beschränken wir uns auf das mittelfristig Machbare.

Eine Transnationale Germanistik hat es bei der Behandlung ihrer Gegenstände genuin mit Brechungen, ja im systematischen Sinne mit Negationen zu tun. Diese Erkenntnisbewegung der Negation wurde in den letzten Jahren verschieden gesehen und benannt: das Andere, das Fremde, das Außen, auch das Inter- und so weiter - und sie ist eben auf dieser Tagung scharfsichtig zum Thema erhoben worden.

Nimmt man diese Eigenheit der Wissenschaft ernst, so ist im erkenntnismethodischen Sinne Kritik ihr Triebmittel - und nicht etwa Selbstvergewisserung oder bloßer Ausbau. Das hat Konsequenzen für die innere Organisation dieser Wissenschaft und für den lehr- und Forschungsaustausch.

Eine Transnationale Germanistik - und dessen war sich die Germanistik als solche schon länger bewusst - kann hier oder irgendwo nicht einfach auf kanonisierte Gegenstände oder auf fraglose Wissensbestände zurückgreifen. Meines Erachtens ist dies kein kontingentes Phänomen; doch diese Diskussion gehört in einen wissenschaftstheoretischen Disput. Wissenschaftspraktisch besteht die Konsequenz aus der genannten Situation darin, dass sich die Lehrgegenstände in der (Transnationalen) Germanistik nicht umstandslos in gleichartige Lehrpläne umsetzen lassen, dass nicht-kanonisierte Inhalte vielfältige Vermittlungsformen zulassen, ja geradezu herausfordern. Den europäischen Vorgaben von Bologna, die eindeutige und homogene ECTSPunktvergaben fordern, widerspricht eine derartige innere Struktur allerdings zutiefst.

Insofern müsste eine Praxis unserer germanistischen Wissenschaften darin bestehen, die Vielfalt auch in dieser Hinsicht zu bewahren und positiv zu wenden. Freilich setzt das eine kritische Selbstbesinnung darüber voraus, wozu und unter welchen Bedingungen das Forschungsspektrum und die Studieninhalte am gegebenen Ort so sind, wie sie sind, wobei der Frage, ob sie den je besonderen gesellschaftlichen und institutionellen Voraussetzungen sowie den Erfahrungen der Studierenden angemessen sind, große Bedeutung zukommt. Vor diesem Hintergrund könnten - auf der Basis wissenschaftlicher Mindeststandards - die jeweils landes- oder kulturspezifischen Differenzen der Inhalte und Vermittlungsmethoden (auf Deutsch!) in die vielgepriesene Konkurrenz zueinander treten und je eigene Leistungskriterien erarbeitet werden.

Ein wechselseitiger Austausch in der Lehre - durch verbindliche Praktika und durch ausländische Studiensemester - würde mithin auch einen Aus- 
tausch in Lehrverfahren und Lehrgegenständen einschließen, der im Wege des Dozentenaustauschs untermauert werden könnte. All dies setzt voraus, dass sich die Vertreter der Germanistiken - beispielsweise vermittelt über die jeweiligen Germanistikverbände - zu planenden und beratenden Gruppen zusammenfinden. Das möchte ich auch auf Forschungskooperationen ausdehnen, die im nationalen, europäischen wie internationalen Bildungsrahmen Unterstützung finden müssten.

Kurzfristig sind m.E. vergleichende empirische Begleitforschungen $\mathrm{zu}$ Lern- und Wissenschaftskulturen anzuregen. Vielleicht erweist sich das alte Humboldtsche Konzept des forschenden Lernens und lehrenden Forschens dann doch nicht mehr als so ineffektiv, wie dies unsere Ministerien in Deutschland glauben machen wollen.

Mittelfristig wären innerhalb der germanistischen Linguistik konkrete empirische Studien zu je sprachkontrastiven bzw. sprachvergleichenden Zugängen zur deutschen Sprache unter den Bedingungen von Fremd- oder ZweitSprachigkeit, von Zwei- und Mehrsprachigkeit in einer Art Netzstruktur erstrebenswert. Sie sollten sich sowohl auf die konkreten Situationen des Spracherwerbs als auch auf die sprachtheoretisch geleitete wissenschaftliche Befassung mit dem Deutschen beziehen. Fragen zu differenten grammatischen Traditionen und zu einem eher praktischen oder eher analytischen Umgang mit dem Gegenstand Sprache wären zum Beispiel als Einzelfragen von Interesse.

Längerfristig erwarte ich durch eine Konzertierung der linguistischen Forschungen vor allem eine Beförderung empirischer Untersuchungen zu differenten sprachlichen Handlungspraxen, die sowohl für eine Komparatistische Pragmatik und Angewandte Diskurs- und Textforschung des Deutschen als auch für wirkliche Interkulturalität den Kenntnisstand aus den nationalen Dimensionen herausholt.

Im besonderen ließe sich durch eine solche Art von internationaler (!) Forschung eine Komparatistik der institutionellen Kommunikation vorantreiben, jeweils bezogen auf das Deutsche. Primäres Interesse gälte gewiss den ökonomischen sowie den medizinischen oder juristischen Institutionen. Die zu behandelnden Brechungen erfolgen hier (a) durch autochthone institutionelle Kommunikation in Deutsch als Erst- oder als Fremdsprache bzw. professionell oder laienhaft gedolmetscht, (b) durch eine Kommunikation auf Deutsch in Institutionen nicht-deutschsprachiger Länder und (c) durch institutionelle Kommunikation in den je anderen (Herkunft-) Sprachen und Kulturen im Vergleich. Dazu ist freilich eine enge Kooperation mit den jeweils einschlägigen Einzel-Sprachwissenschaften und mit den Wissenschaften und Institutionen des Untersuchungsfeldes erforderlich. Eine vergleichende Pragmatik der institutionellen Kommunikation könnte neben den linguistischen Erkenntnissen zugleich landeskundliche Kenntnisse befördern und für eine interkulturelle Landeskunde fruchtbar werden. Schließlich wären gerade diese gesellschaftlichen Bereiche in ihrem Reflex in der Literatur zu betrachten. 
Dies waren nur einige Beispiele einer transnational konzertierten Lehre und Forschung, die ihre Fragestellungen und methodischen Anregungen durch einen beständigen Diskurs zwischen den Disziplinen der Länder beziehen könnte.

\section{Universitätsstrukturen für eine Transnationale Germanistik}

Abschließend nur wenige Worte zur Alltagspraxis eines solchen Gesamtkonzeptes, insbesondere zur universitären Struktur.

Es sollte deutlich geworden sein, dass eine Transnationale Germanistik wie bereits seit längerem die Fächer Deutsch als Fremd- und als Zweitsprache - ein genuin interdisziplinäres und auf den kritischen wissenschaftlichen Diskurs angewiesenes Unternehmen ist. Zudem sollte das Verhältnis von Theorie und Praxis ein durch Praktika und Feldforschungen wechselseitig abgesichertes sein. Dies stellt die Wissenschaftlichkeit nicht zur Disposition; vielmehr handelt es sich um eine komplexe akademische und also universitäre Disziplin, die auf allgemeine Problemlösungen hin ausbildet und nicht auf spezifische Berufsfelder vorbereitet, wie dies - in der BRD zumindest - die Aufgabe von Fachhochschulen ist. Bildungspolitik und Forschungsförderung müssen dies zur Kenntnis nehmen.

Die angesprochene Interdisziplinarität bezieht sich genuin auf alle anderen Philologien bzw. Sprach- und Literaturwissenschaften sowie auf die Allgemeine und vergleichende Sprachwissenschaft und die literaturwissenschaftliche Komparatistik. Darüber hinaus sind weitere Verbindungen zu ziehen, die hier nicht anzuführen sind.

Vielmehr möchte ich an dieser Stelle an ein Innovationsprojekt erinnern, das vor gut dreißig Jahren - etwa zu Beginn meines Studiums - in der BRD entwickelt wurde und zu einem Memorandum in dem kleinen westfälischen Städtchen Rheda führte. Ich denke an die wechselseitig angelegten Konzepte einer sprachübergreifenden, ja mehrsprachigen Literaturwissenschaft und Linguistik von Wolfgang Iser und Harald Weinrich. Sie ebenso wie das Rhedaer Memorandum sind in dem bekannten Band „Ansichten einer künftigen Germanistik" von Jürgen Kolbe (1969) abgedruckt. Diese Konzepte sehen keine Trennungen zwischen z. B. der germanistischen, anglistischen, romanistischen und slavistischen Linguistik vor und setzen bei den Lehrenden wie Lernenden mindesten die fundierte Kenntnis zweier moderner Fremdsprachen voraus. Für ein mehrsprachiges Europa dürfte darin gewiss ein besonderer Reiz liegen.

Nun sind die Konzepte seitdem im wesentlichen an zwei Reformuniversitäten durchgeführt worden, in Konstanz und in Bielefeld. Eine andere Umsetzungsform wurde z. B. mit der Einrichtung der Allgemeinen Sprachwissenschaft - damals auch als Schulfach in NRW - neben den traditionellen Philologien an der Universität Düsseldorf erprobt, wobei sich die Allgemei- 
ne Linguistik in den letzten Jahrzehnten allerdings in eine universalistische und damit dezidiert von den Einzellinguistiken wegführende Richtung entwickelte. Die Bielefelder und Konstanzer „Lili-Fakultäten“ gelten ihrerseits seit einigen Jahren als gescheitert, wiewohl die Studentenzahlen hoch sind. Die negative Einschätzung hat nicht zuletzt damit zu tun, dass der herangezogene wissenschaftliche Nachwuchs keine derart breite Arbeitsmöglichkeit anderswo fand und also in die Einzeldisziplinen zurückkehrte und dass die sprachlichen Voraussetzungen nicht immer hinreichend gegeben waren. Zudem war das Konzept an damalige inhaltliche Vorgaben gebunden, die sich theoretisch und methodisch teilweise diversifiziert und auseinanderentwickelt haben.

Trotz alledem halte ich es für sinnvoll, heute vor einem entfalteten Forschungsstand und insbesondere - das ist gegenüber damals ein Novum - mit inzwischen breit akademisch entwickeltem Kenntnisstand in Deutsch als Fremdsprache und Deutsch als Zweitsprache in eine ähnliche Richtung weiterzudenken. Das Konzept einer Transnationalen Germanistik könnte einen Baustein dazu abgeben.

\section{Literatur}

Abraham, Werner (in diesem Band): Faszination der kontrastiven Linguistik DaF: der Parameter ,schwere/leichte" Sprache unter typologischer Sicht.

Anderson, Benedict (1983, dtsch. 1988): Die Erfindung der Nation. Zur Karriere eines erfolgreichen Konzepts. Frankfurt/M.: Campus.

Aufruf zur Begründung eines Deutschen Germanisten-Verbandes (1912) In: Reiss, Gunter (Hg.) (1973): Materialien zur Ideologiegeschichte der deutschen Literaturwissenschaft. Tübingen: Niemeyer. Bd. 1, S. 82-83.

Bierwisch, Manfred (1993): Ludwig Jägers Kampf mit den Windmühlen - Anmerkungen zu einer merkwürdigen Sprach(wissenschafts)verwirrung. In: Zeitschrift für Sprachwissenschaft 12.1, S. 107-112.

Bourdieu, Pierre (1988): homo academicus. Frankfurt: Suhrkamp.

Bühler, Karl $\left(1934 ; 1965^{2}\right)$ : Sprachtheorie. Jena/Stuttgart: Fischer.

Cherubim, Dieter (1984): Sprachgeschichte im Zeichen der linguistischen Pragmatik. In: Besch, W./Reichmann, O./Sonderegger, St. (Hg.): Sprachgeschichte. Ein Handbuch. Berlin: de Gruyter. Bd. 1, 802-815 (2. vollst. neu bearb. u. erw. Aufl. 1998, S. 538-551).

Coulmas, Florian (1999): Language masters: defying linguistic determinism. In: International Journal of the Sociology of Language 137, S. 27-39.

Durell, Martin (in diesem Band): Register, Variation und Fremdsprachvermittlung.

Ehlich, Konrad (1981): Sprachmittel und Sprachzwecke. Antrittsvorlesung Universität Düsseldorf. (= Tilburg Papers in Language and Literature TILL 1/1982).

Ehlich, Konrad (1996): Sprache als System versus Sprache als Handeln. In: Dascal, Marcello et al. (Hg.): HSK „Sprachphilosophie“. Berlin: de Gruyter. S. 952-963.

Ehlich, Konrad (1996): Interkulturelle Kommunikation. In: Nelde, Peter H. et al. (Hg.): Kontaktlinguistik. Ein Handbuch (HSK 15.1). Berlin: de Gruyter. S. 920-931.

Ehlich, Konrad (2000): Europäische Sprachen im Zeitalter der Postnationalität. In: Kugler/Redder. (Hg.), S. 186-195.

Fabricius-Hansen, Cathrine (1999): Grammatik und Verstehen. In: Redder/Rehbein. (Hg.), S. 15-29. 
Fabricius-Hansen, Cathrine (in diesem Band): Deutsch, eine „reife“ Sprache - Ein Plädoyer für die Komplexität.

Gardt, Andreas: (1999) Geschichte der Sprachwissenschaft in Deutschland. Vom Mittelalter bis ins 20. Jahrhundert. Berlin: de Gruyter.

Gardt, Andreas (Hg.) (2000): Nation und Sprache. Die Diskussion ihres Verhältnisses in Geschichte und Gegenwart. Berlin: de Gruyter.

Garlin, Edgardis (2000): Bilingualer Erstspracherwerb. Sprachlich Handeln, Sprachprobieren, Sprachreflexion. München: Verlag für Sprache und Sprachen.

Georges, H. (ND 1988): Ausführliches Lateinisch-Deutsches Handwörterbuch. 2 Bde. Darmstadt: Wissenschaftliche Buchgesellschaft.

Glück, Helmut (Hg.) (2002): Geschichte des Deutschen als Fremdsprache. Bd. 3. Berlin: de Gruyter.

Grewendorf, Günther (1993): Der Sprache auf der Spur: Anmerkungen zu einer Linguistik nach Jäger Art. In: Zeitschrift für Sprachwissenschaft 12.1, S. 113-132.

Henne, Helmut (1980): Probleme einer historischen Gesprächsanalyse. In: Sitta, H. (Hg.): Ansätze zu einer pragmatischen Sprachgeschichte. Tübingen: Niemeyer. S. 89-102.

Hinnenkamp, Volker (1994): Interkulturelle Kommunikation. (Studienbibliographie Sprachwissenschaft 11) Heidelberg: Groos.

Hoffmann, Ludger (1995): „Gegenstandskonstitution“ und „Gewichtung“: Eine kontrastiv-grammatische Perspektive. In: Jahrbuch DaF 21, S. 235-264.

Homburger Empfehlungen zur Förderung der Europäischen Hochsprachen (2001). In: Ehlich, K./ Ossner, J./ Stammerjohann, H. (Hg.): Hochsprachen in Europa. Freiburg/ B.: fillibach. S. 387-389.

Humboldt, Wilhelm von (ND1963): Schriften zur Sprachphilosophie. Bd. III. Darmstadt: Wissenschaftliche Buchgesellschaft. (darin: Ueber die Verschiedenheit des menschlichen Sprachbaues und ihren Einfluss auf die geistige Entwicklung des Menschengeschlechts [1830-1835]).

Iser, Wolfgang (1969): Überlegungen zu einem literaturwissenschaftlichen Studienmodell. In: Kolbe (Hg.), S. 193-207.

Jäger, Ludwig (1993): „Language, what ever that may be.“ - Die Geschichte der Sprachwissenschaft als Erosionsgeschichte ihres Gegenstandes. In: Zeitschrift für Sprachwissenschaft 12.1, S. 77-106.

Janota, Johannes (Hg.) (1980): Eine Wissenschaft etabliert sich: 1810-1870. (= Texte zur Wissenschaftsgeschichte der Germanistik III) Tübingen: Niemeyer.

Kallmeyer, Werner (Hg.) (1994): Exemplarische Analysen des Sprachverhaltens in Mannheim. Berlin: de Gruyter.

Knobloch, Clemens (1988): Geschichte der psychologischen Sprachauffassung in Deutschland von 1850 bis 1920 . Tübingen: Niemeyer.

Kolbe, Jürgen (Hg.) (1969; 4. rev. Aufl. 1970): Ansichten einer künftigen Germanistik. München: Hanser.

Koole, Tom/ten Thije, Jan (1994): The construction of intercultural discourse. team discussions of educational advisers. Amsterdam: Rodopi.

Krumm, Hans-Jürgen (in diesem Band): Deutsch von außen - in der Inlandsgermanistik.

Krusche, Dietrich/Wierlacher, Alois (Hg.) (1990): Hermeneutik der Fremde. München: iudicium.

Kugler, Hartmut/Redder, Angelika (Hg.) (2000): Euro-Deutsch.Kontroversen um die Deutschsprachigkeit im europäischen Mehrsprachenraum. (= Mitteilungen des Deutschen Germanistenverbandes 47, H. 2-3). Bielefeld: Aisthesis.

Kugler, Helmut et al. (Hg.) (im Druck): www.Germanistik2001.de. Deutscher Germanistentag in Erlangen 2001. Bielefeld: Aisthesis. 
Lämmert, Eberhard (1967): Germanistik - eine deutsche Wissenschaft? In: Lämmert, Eberhard/Killy, Walther/Conrady, Carl Otto/Polenz, Peter von (Hg.): Germanistik eine deutsche Wissenschaft. Frankfurt/M.S. 7-41.

Maas, Utz (1996): Verfolgung und Auswanderung deutschsprachiger Sprachforscher 1933-1945. Bd. 1. Osnabrück: secolo.

Mannheimer Florentiner Empfehlungen zur Förderung der europäischen Hochsprachen. In: Stickel, Gerhard (Hg.) (2002): Europäische Hochsprachen und mehrsprachiges Europa. Mannheim: Institut für Deutsche Sprache. S. 230 -232.

Meisel, Jürgen (2000): The Simultaneous Acquisition of Two First Languages: Early Differentiation and Subsequent Development of Grammars. Hamburg: Arbeiten zur Mehrsprachigkeit, Folge B 7.

Mertens, Volker (Hg.) (1988): Die Grimms, die Germanistik und die Gegenwart. Wien: Fassbaender (darin: Rautenberg, Ursula: Germanistik als Wissenschaft - Aspekte zur Geschichte des Fachs im frühen 19. Jahrhundert. S. 25-48).

Moll, Melanie (2001): Das wissenschaftliche Protokoll. Vom Seminardiskurs zur Textart. München: iudicium.

Müller, Jörg Jochen (Hg.) (1974, repr. 2000): Germanistik und deutsche Nation 1806-1848. Zur Konstitution bürgerlichen Bewusstseins. Stuttgart: Metzler.

Müller, Natascha/Cantone, Katja/Kupisch, Tanja/Schmitz, Katrin (2001): Das mehrsprachige Kind: Italienisch - Deutsch. Hamburg: Arbeiten zur Mehrsprachigkeit. Folge B 16.

Panzer, Friedrich (1973): Grundsätze und Ziele des Deutschen Germanisten-Verbandes (1912). In: Reiss, Gunter (Hg.) (1973): Materialien zur Ideologiegeschichte der deutschen Literaturwissenschaft. (= Texte zur Wissenschaftsgeschichte der Germanistik IV) Tübingen: Niemeyer. Bd. 1, S. 83-91.

Polenz, Peter von (1994-20002): Deutsche Sprachgeschichte. 3 Bde. Berlin: de Gruyter (Bd. I: überarb. u. erw. 2000²; Bd.II: 1994, Bd. III: 1999).

Redder, Angelika (Hg.) (2002): „Effektiv studieren“. Texte und Diskurse in der Universität. (= OBST-Beiheft 12) Duisburg: Gilles/Francke.

Redder, Angelika/Rehbein, Jochen (1987): Zum Begriff der Kultur. In: dies. (Hg.): Arbeiten zur interkulturellen Kommunikation. Osnabrück: OBST 38, S. 7-21.

Redder, Angelika/Rehbein, Jochen (Hrsg.) (1999): Grammatik und mentale Prozesse. Tübingen: Stauffenburg.

Rehbein, Jochen (1995): Grammatik kontrastiv - am Beispiel von Problemen mit der Stellung finiter Elemente. In: Jahrbuch DaF 21, S. 265-292.

Rehbein, Jochen/Grießhaber, Wilhelm (1996): L2-Erwerb versus L1-Erwerb: Methodologische Aspekte ihrer Erforschung. In: Ehlich, K. (Hg.): Kindliche Sprachentwicklung. Opladen: Westdeutscher Verlag. S. 67-119.

Rhedaer Memorandum (1969): Memorandum zur Reform des Studiums der Linguistik und der Literaturwissenschaft. In; Kolbe (Hg.), S. 217-220.

Römer, Ruth (1985): Sprachwissenschaft und Rassenideologie in Deutschland. München: Fink (2. verb. Aufl. 1989)

Rompeltien, Bärbel (1994): Germanistik als Wissenschaft. Zur Ausdifferenzierung und Integration einer Fachdisziplin. Opladen: Westdeutscher Verlag.

Schildt, Joachim (1976): Abriß der Geschichte der deutschen Sprache. Berlin (DDR): Akademie-Verlag.

Schramm, Karen (2001): L2-Leser in Aktion. Der sprachliche Leseprozess als mentales Handeln. Münster: Waxmann.

Tutzinger Thesen zur Sprachenpolitik in Europa. In: Kugler/Redder (Hg.) (2000), S. 293-296. 
Tracy, Rosemarie (2000): Language Mixing as a Challenge to Linguistics. In: Döpke, S. (ed.): Cross-linguistic structures in simultaneous bilingualism. Amsterdam: Benjamins. S. 11-36.

Wegener, Philipp (1885): Untersuchungen über die Grundfragen des Sprachlebens. Halle (ND von C. Knobloch, Amsterdam: Benjamins).

Weinrich, Harald (1969): Überlegungen zu einem Studienmodell der Linguistik. In: Kolbe (Hg.), S. 208-216.

Weinrich, Harald (1986): Klammersprache Deutsch. In: Sprachnormen in der Diskussion. Beiträge vorgelegt von Sprachfreunden. (G. Drosdowski zum 15.10.1986) Berlin: de Gruyter. S. 116-145.

Weinrich, Harald (1993): Textgrammatik der deutschen Sprache. (unter Mitarbeit von Maria Thurmair, Eva Breindl, Eva-Maria Willkop) Mannheim: Duden.

Wiesmann, Bettina (1999): Mündliche Kommunikation im Studium. München: iudicium.

Zifonun, Gisela/Hoffmann, Ludger/Strecker, Bruno et al. (1997): Grammatik der deutschen Sprache. 3 Bde. Berlin: de Gruyter.

Zifonun, Gisela (in diesem Band): Deutsch im Spiegel der europäischen Sprachen. 